\title{
Transcriptional Regulation of Human NANOG by Alternate Promoters in Embryonic Stem Cells
}

\author{
Satyabrata Das ${ }^{1}$, Snehalata Jena ${ }^{1}$, Eun-Mi Kim¹, Nicholas Zavazava ${ }^{1}$ and Dana N. Levasseur ${ }^{1,2 *}$
}

${ }^{1}$ Department of Internal Medicine, Roy J. and Lucille A. Carver College of Medicine, lowa City, USA

${ }^{2}$ Programs in Genetics and Molecular and Cellular Biology, University of lowa, lowa City, lowa 52242, USA

\begin{abstract}
Introduction: The potential of pluripotent stem cells to be used for cell therapy depends on a comprehensive understanding of the molecular mechanisms underlying their unique ability to specify cells of all germ layers while undergoing unlimited self-renewal. Alternative splicing and alternate promoter selection contribute to this mechanism by increasing the number of transcripts generated from a single gene locus and thus enabling expression of novel protein variants which may differ in their biological role. The homeodomain-containing transcription factor NANOG plays a critical role in maintaining the pluripotency of Embryonic Stem Cells (ESC). Therefore, a thorough understanding of the transcriptional regulation of the NANOG locus in ESCs is necessary.
\end{abstract}

Methods: Regulatory footprints and transcription levels were identified for NANOG in human embryonic stem cells from data obtained using high-throughput sequencing methodologies. Quantitative real-time PCR following reverse transcription of RNA extracted human ESCs was used to validate the expression of transcripts from a region that extends upstream of the annotated NANOG transcriptional start. Promoter identification and characterization was performed using promoter reporter and electrophoretic mobility shift assays.

Results: Transcriptionally active chromatin marking and transcription factor binding site enrichment were observed at a region upstream of the known transcriptional start site in NANOG. Expression of novel transcripts from this transcriptionally active region confirmed the existence of NANOG alternative splicing in human ESCs. We identified an alternate NANOG promoter of significant strength at this upstream region. We also discovered that NANOG autoregulates its expression by binding to its proximal downstream promoter.

Conclusion: Our study reveals novel transcript expression from NANOG in human ESCs, indicating that alternative splicing increases the diversity of transcripts originating from the NANOG locus and that these transcripts are expressed by an alternate promoter. Alternative splicing and alternate promoter usage collaborate to regulate NANOG, enabling its function in the maintenance of ESCs.

Keywords: NANOG; Alternative splicing; Alternate promoter; Pluripotency; Self-renewal; Differentiation; Stem cells

\section{Introduction}

Embryonic Stem Cells (ESCs), derived from the inner cell mass of the mammalian blastocyst, hold great promise for future therapeutic applications because of their unique ability to both proliferate indefinitely (self-renewal) in an undifferentiated state and retain the potential to give rise to every cell type in the body (pluripotency) [13]. Differentiated somatic cells can be reprogrammed into ESC-like Induced Pluripotent Stem cells (iPSCs) using various mixtures of pluripotency factors $[4,5]$. iPSCs provide a more attractive and patienttailored platform for cell therapy, and proof-of-principle experiments in mice suggest genetic therapies using these cells are effective in ameliorating disease [6]. For iPSC based therapies to reach fruition, a more thorough understanding of the molecular mechanisms that govern the undifferentiated ESC state will be required.

Several recent studies using proteomics, RNA interference, loss of function screens, genome scale location analyses and transcriptome profiling have identified transcription factors that serve as the framework for maintaining ESC pluripotency and self-renewal [7-14]. The transcription factors NANOG, OCT4 and SOX2 constitute a core regulatory network which governs the stable expression of self-renewal factors while keeping gene differentiation programs suppressed in human and mouse ESCs $[7,8,10,11,15]$. Therefore, it is essential to understand the transcriptional properties of these core transcription factors to better enable controlled differentiation of pluripotent stem cells. Alternative splicing (AS) and alternate promoter usage adds another layer of regulatory control to the already complex process of pluripotency maintenance and differentiation. AS can vastly increase proteomic diversity by the addition or deletion of protein domains. These changes in coding sequence frequently lead to alterations in protein structure or subcellular localization, resulting in altered protein-protein interactions and biological outputs. AS mediated modifications of non-coding sequences can alter post-transcriptional regulation by mechanisms that include alternate 3' untranslated region (UTR) usage and the associated revision of microRNA binding footprints [16]. Indeed ESCs express a large diversity of splice isoforms that implies switching from pluripotency to lineage commitment and differentiation involves AS and alternate promoter selection [17-24].

The homeobox-containing core transcription factor NANOG is specifically expressed in pluripotent cells of the mouse preimplantation embryo, embryonic germ (EG) cells and ESCs of murine [25-27] and

*Corresponding author: Dana N. Levasseur, 285 Newton Road, Department of Internal Medicine, Roy J. and Lucille A. Carver College of Medicine, lowa City, IA, 52242 USA, Tel: 319-384-4686; Fax: 319-3535552; E-mail: dana-levasseur@uiowa.edu

Received July 18, 2012; Accepted August 22, 2012; Published August 24, 2012

Citation: Das S, Jena S, Kim EM, Zavazava N, Levasseur DN, et al. (2012) Transcriptional Regulation of Human NANOG by Alternate Promoters in Embryonic Stem Cells. J Stem Cell Res Ther S10:009. doi:10.4172/2157-7633.S10-009

Copyright: (c) 2012 Das S, et al. This is an open-access article distributed under the terms of the Creative Commons Attribution License, which permits unrestricted use, distribution, and reproduction in any medium, provided the original author and source are credited. 
Citation: Das S, Jena S, Kim EM, Zavazava N, Levasseur DN, et al. (2012) Transcriptional Regulation of Human NANOG by Alternate Promoters in Embryonic Stem Cells. J Stem Cell Res Ther S10:009. doi:10.4172/2157-7633.S10-009

human origin [28]. Disruption of NANOG leads to differentiation of ESCs into extraembryonic cell lineages in both murine $[9,25,26]$ and human $[29,30]$ ESCs. Overexpression of NANOG confers LIF (leukemia inhibitory factor)-independent self-renewal in mouse ESCs [26], and enables feeder-free propagation in human ESCs [31]. We recently reported an upstream extension of the mouse Nanog gene which enables production of the novel Nanog protein variants Nanog $\mathrm{b}$ and $\operatorname{Nanog} \mathrm{c}$ that exhibit altered capacities for self-renewal and pluripotency in ESCs [23]. Another recent study has also reported a similar AS event in the human NANOG gene in embryonal carcinoma cells from an upstream region at the 5 ' region, resulting in additional transcripts and a protein variant that initiates from a downstream methionine [32] and is the human ortholog of mouse Nanog c [23].

In the present study, we have verified the existence of novel alternate NANOG transcripts in human ESCs. We have identified a strong alternate promoter upstream of the novel transcripts using a neomycin resistance reporter assay that enables promoter strength to be assessed on chromatinized templates. The core transcription factors OCT4 and SOX2 have been shown to activate NANOG expression by binding to cis-regulatory elements in the proximal NANOG promoter [33], whereas Kruppel-like zinc-finger transcription factor KLF4 and the homeodomain containing transcription factor PBX1 also activate the NANOG promoter in cooperation with OCT4 and SOX2 [34]. We also demonstrate here that Nanog participates in positive autoregulation of its own proximal promoter.

\section{Materials and Methods}

\section{Cell culture}

Mouse ESC lines (CJ7 or J1) were maintained on gelatin-coated plates in a feeder-free condition as described previously in standard ESC media supplemented with LIF $[35,36]$. Human ESCs (H13, from WiCell) were cultured in DMEM/F12 medium supplemented with 20\% Knockout Serum Replacement (GIBCO/BRL), 10 ng/ml bFGF, $1 \mathrm{mM}$ GlutaMax, $50 \mathrm{U} / \mathrm{ml}$ penicillin and $50 \mu \mathrm{g} / \mathrm{ml}$ streptomycin, $1 \mathrm{X}$ nonessential amino acids and $100 \mu \mathrm{M} 2$-mercaptoethanol (Invitrogen) on top of $\gamma$-irradiated MEFs. Pluripotent ESCs were sorted from differentiated ESCs and MEFs using Pluripotent Stem Cell microbeads (Miltenyi Biotec).

\section{Plasmid construction}

An EF1a-Flag-Biotin expression plasmid used previously [7] was adapted to analyze the ability of NANOG promoter fragments to drive the expression of a neomycin phosphotransferase coding sequence and impart neomycin resistance $\left(\mathrm{Neo}^{\mathrm{R}}\right)$. For this, the EFla-FlagBiotin sequence was removed and a Gateway recombination cassette (Invitrogen) was ligated in its place to generate a gateway-adapted plasmid. The $\mathrm{Neo}^{\mathrm{R}}$ cassette was ligated downstream of the gateway cassette followed by a polyadenylation (polyA) signal from the Nanog 3' UTR. Different fragments of the NANOG promoter were amplified from HEK/293T cell genomic DNA with attB site containing primers (Table 1). The PCR product was recombined into the pDONR221 entry vector by Gateway BP reaction and the promoter sequences were then transferred to the Gateway-adapted $\mathrm{Neo}^{\mathrm{R}}$ destination vector by a Gateway LR reaction, followed by sequence verification.

For the luciferase reporter, the polyA sequence downstream of the firefly luciferase in the pGL3-basic plasmid (Promega) was replaced with the polyA signal from the Nanog 3' UTR followed by Gateway adaptation of the plasmid by ligating a Gateway cassette into the MCS to generate the firefly luciferase destination vector. Different NANOG promoters were then PCR amplified with attB sites and recombined into the firefly luciferase destination vector by sequential BP-LR reactions as described above. Site-directed mutagenesis (Stratagene) was used to mutate the different transcription factor binding sites that were then verified by sequencing.

\section{Neomycin resistance assay}

Linearized plasmids $(5 \mu \mathrm{g}$ each) containing different NANOG promoter fragments driving the $\mathrm{Neo}^{\mathrm{R}}$ expression cassette were trasfected into $1 \times 10^{6} \mathrm{~J} 1$ ESCs using Lipofectamine (Invitrogen) in 6-well plates. Transfected cells were selected with $200 \mu \mathrm{g} / \mathrm{ml} \mathrm{G}-418$ $48 \mathrm{hr}$ post-transfection for 8 days and stable colonies were counted. Assays were done in duplicate and were from at least two independent transfections.

\begin{tabular}{|c|c|c|}
\hline Usage & Primers Used (5'-3') & Amplimer size \\
\hline $\begin{array}{l}\text { Promoter } \\
\text {-Reporter }\end{array}$ & $\begin{array}{l}\text { hsNgProm -2401-AttB1-F1: ggggACAAGTTTGTACAAAAAAGCAGGCTTAtctctcatgcctttacccaaa } \\
\text { hsNgProm -1817-AttB1-F1: ggggACAAGTTTGTACAAAAAAGCAGGCTTaaaaatctaaagtcagatagcttcc } \\
\text { hsNgProm -1788-AttB1-F1: ggggACAAGTTTGTACAAAAAAGCAGGCTTAcctcaactttattccaattgcttt } \\
\text { hsNgProm -1737-AttB2-R1: ggggACCACTTTGTACAAGAAAGCTGGGTAggccgacttactacattcttcg } \\
\text { hsNgProm -1702-AttB2-R1: ggggACCACTTTGTACAAGAAAGCTGGGTAgtgaaagaccaaagggaagg } \\
\text { hsNgProm -258-AttB1-F1: ggggACAAGTTTGTACAAAAAAGCAGGCTTAtcccattcctgttgaaccat } \\
\text { hsNgProm +34-AttB2-R1: ggggACCACTTTGTACAAGAAAGCTGGGTAaacgttaaaatcctggagtctct } \\
\text { Hoxb6 Prom-AttB1-F1: ggggACAAGTTTGTACAAAAAAGCAGGCTTaggttgataggtttgtgcgc } \\
\text { Hoxb6 Prom-AttB2-R1: ggggACCACTTTGTACAAGAAAGCTGGGTAccgggtttatgatttgttgtgt }\end{array}$ & \\
\hline qRTPCR & $\begin{array}{l}\text { NANOG F3: tgtgggcctgaagaaaactatc } \\
\text { NANOG R3: gctgtcctgaataagcagatcc } \\
\text { NANOG F1: tcctttggtcttcactcc } \\
\text { NANOG R1: ctcctgtcccattgtgtct } \\
\text { NANOG F2: tatgcaaagacccccttctg } \\
\text { NANOG R2: gctctccaaagggcaggta } \\
\text { GAPDH F1: tgggtgtgaaccatgagaagta } \\
\text { GAPDH R1: gagtccttccacgataccaaag }\end{array}$ & $\begin{array}{l}11 \mathrm{bp} \\
55 \mathrm{bp} \\
58 \mathrm{bp} \\
125 \mathrm{bp}\end{array}$ \\
\hline
\end{tabular}

Table 1: Primers used in the Study. For the following promoter primers the attB site sequences are shown in capital letters. Information for EMSA primers can be found in Figure 5. 


\section{Luciferase reporter assay}

The following constructs were cotransfected into $5 \times 10^{5}$ CJ7 ESCs in 12-well plates: $2 \mu \mathrm{g}$ of firefly luciferase reporter and $50 \mathrm{ng}$ of the renilla luciferase vector (pRL-Null, Promega). 48 hrs post-transfection lysates were harvested for luciferase assays. Luciferase activity was measured by the Dual-Luciferase reporter assay system (Promega) using a BioTek Synergy 4 microplate reader. The firefly luciferase activity was normalized to the renilla activity to compensate for transfection variabilities in different wells. At a minimum, triplicate measurements were taken from two independent transfections.

\section{Electrophoretic mobility shift assay (EMSA)}

Nuclear extracts were prepared from CJ7 ESCs as described previously $[37,38]$. Briefly, cells were harvested by trypsinization and washed twice with ice-cold phosphate-buffered saline and once with 5 pellet volumes of buffer A (20 mM Hepes $\mathrm{pH} 7.9,10 \mathrm{mM} \mathrm{KCl}, 1 \mathrm{mM}$ EDTA, $1 \mathrm{mM} \mathrm{Na} \mathrm{VO}_{4}, 10 \%$ (vol/vol) glycerol freshly supplemented with $1 \mathrm{mM}$ DTT, $1 \mathrm{mM}$ PMSF, $1 \%$ protease inhibitor cocktail (Sigma)). Cell pellets were then incubated on ice for 10 min with 5 pellet volumes of buffer A and followed by lysis with 20 strokes in a Dounce homogenizer. Following lysis, pelleted nuclei were resuspended in 3 pellet volumes of buffer B (20 mM Hepes pH 7.9, $10 \mathrm{mM} \mathrm{KCl,} 350$ $\mathrm{mM} \mathrm{NaCl}, 1 \mathrm{mM}$ EDTA, 1 mM Na $\mathrm{VO}_{4}, 20 \%$ (vol/vol) glycerol) freshly supplemented with $1 \mathrm{mM}$ DTT, $1 \mathrm{mM}$ PMSF, $1 \%$ protease inhibitor cocktail (Sigma)) and incubated at $4^{\circ} \mathrm{C}$ with rotation for $30 \mathrm{~min}$. After that, the nuclear lysate was centrifuged and the supernatant containing the nuclear extract was used in the EMSA or stored at $-80^{\circ} \mathrm{C}$.

For EMSA, complementary oligonucleotides having an additional $\mathrm{G}$ at their respective 5 '-ends were annealed in annealing buffer $(10 \mathrm{mM}$ Tris-Cl, pH 7.5, $1 \mathrm{mM}$ EDTA, and $100 \mathrm{mM} \mathrm{NaCl}$ ) and the resulting double-stranded (ds) oligonucleotides were end-filled with $\left[\gamma^{-32} \mathrm{P}\right]$ dCTP using Roche Klenow for 30 minutes at $37^{\circ} \mathrm{C}$. Following this, the radio-labeled ds-oligonucleotides were purified using Roche spin columns. For DNA binding reactions, $6 \mu \mathrm{g}$ of nuclear extract was added to a $20 \mu \mathrm{l}$ reaction containing $400 \mathrm{fM}$ of radio-labeled oligonucleotide and $1 \mu \mathrm{g}$ of poly(dIdC) (Roche) in the binding buffer (12 mM Hepes $\mathrm{pH} 7.9,50 \mathrm{mM} \mathrm{KCl}, 1 \mathrm{mM}$ EDTA, 5\% glycerol, $1 \mathrm{mM} \mathrm{DTT}$ ) and incubated on ice for 45 minutes. Where specified, $60 \mathrm{pM}$ of unlabeled double-stranded competitor was included along with the radio-labeled oligonucleotide. Complexes were separated on pre-run $6 \%$ native polyacrylamide gels for $4 \mathrm{hrs}$ at $150 \mathrm{~V}$ in $0.5 \mathrm{X}$ Tris-borate-EDTA. The gels were subsequently dried and autoradiography was performed.

\section{Sequence Analysis and bioinformatics}

All Nanog DNA sequences are from the most recent genomic builds taken from the NCBI public uatabase (July 2012 freeze). DNA alignments were done with CLUSTALW using the AlignX program from the VectorNTI suite. The DNAseI hypersensitivity (HS) peaks for the human H1 and H7 ESCs were obtained from ChIP-Seq data generated by the University of Washington ENCODE group [39]. The histone modification, RNA polymerase 2 and RBBP5 peaks were obtained for the H1 ESCs from the data generated by the Broad Institute/ MGH ENCODE group using ChIP-Seq [40]. The transcription level peaks were obtained the H1 ESCs from data generated by the Wold Lab at Cal Tech, part of the ENCODE consortium assayed by highthroughput sequencing of polyA RNA. The vertebrate conservation measurement tracks were generated using multiz and other tools in the UCSC/Penn State Bioinformatics comparative genomics alignment pipeline. ChIP-Seq binding data for OCT4 and NANOG occupancy were obtained from Kunarso et al. [41] and the P300 ChIP-Seq data were from Lister et al. [42].

For data analysis and peak calling, Fastq files where downloaded from the NCBI SRA and aligned with bowtie 2-2.0.0-beta6 against the ho19 genome using default options. The aligned files where then combined using samtools merge to create one alignment file for each experiment (where multiple fastq files existed). Peak calling was then done with MACS 1.4.2 using default parameters except spacing was changed to 25 and wig file changed to single output. BigWig files were generated with the UCSC tool wigToBigWig.

\section{RNA extraction and quantitative real-time PCR}

RT-PCR (qRT-PCR): RNA was prepared from H13 human ESCs using the PARIS kit (Ambion) following the manufacturer's instructions. An in-column DNAse digestion was performed to remove contaminating genomic DNA. cDNA was synthesized from $1 \mu \mathrm{g}$ of RNA using the SSIII reverse transcriptase (Invitrogen) in a $20 \mu \mathrm{l}$ total volume. cDNAs were diluted 40 -fold and $5 \mu \mathrm{l}$ of the dilute cDNA was used in a $25 \mu \mathrm{l}$ SYBR Green reaction using a Bio-Rad CFX96. Technical replicates were represented from two independent biological replicates. Starting quantities were determined from a GAPDH standard curve.

\section{Results}

\section{Transcriptional regulation of NANOG in human ESCs}

We have recently reported an extended gene structure of the mouse Nanog gene in ESCs [23]. Another study detailed the transcriptional properties of NANOG in a human embryonal carcinoma cell line, revealing additional exons at the 5' end beyond the known gene structure [32]. A comprehensive survey of regulatory DNA within the NANOG locus had not previously been undertaken. We mined data from the ENCODE project and publically available databases to annotate transcriptional regulation of the NANOG gene in human ESCs [39-41]. DNAseI HS is an indicator of active cis-regulatory sequences. The data from two human ESC lines ( $\mathrm{H} 1$ and $\mathrm{H} 7)$ showed strong and identical DNAseI HS positioned $1.8 \mathrm{~kb}$ upstream from the known transcriptional start site (TSS) (Figure 1), whereas a less prominent HS was observed near the TSS. Significant binding enrichment for the basal transcriptional machinery elements represented by RNA polymerase II (POL2), P300 and the transcriptionally permissive chromatin mark histone $\mathrm{H} 3$ lysine 4 trimethylation ( $\mathrm{H} 3 \mathrm{~K} 4 \mathrm{me} 3$ ) were also observed at the $-1.8 \mathrm{~kb}$ region and the known TSS (Figure 1). Binding enrichment for RBBP5, a component of the histone methyltransferase complex was also observed at both regions that were marked by H3K4me3. The ESC core transcription factors OCT4 and NANOG are known to bind the NANOG promoter, and both OCT4 and NANOG binding peaks were observed from the ChIP-Seq data at the $-1.8 \mathrm{~kb}$ region and at the known TSS of NANOG. Transcription levels determined by sequencing of polyA RNA also demonstrate active gene expression at the known TSS and the $-1.8 \mathrm{~kb}$ region in human ESCs (Figure 1). These data, along with the already reported extended gene structure of NANOG in embryonal carcinoma cells, suggest the existence of a similar extension of the NANOG gene in human ESCs that approximates what we have observed for the murine Nanog gene [23].

To confirm the existence of NANOG transcripts originating from the upstream exons in human ESCs as reported in NTERA-2 cells [32] we performed qRT-PCR using RNA from human H13 ESCs. cDNA was synthesized by reverse transcription using oligo (dT) primers to represent fully processed mRNA and qPCR was performed. For detection of transcripts originating from the upstream region, primers 


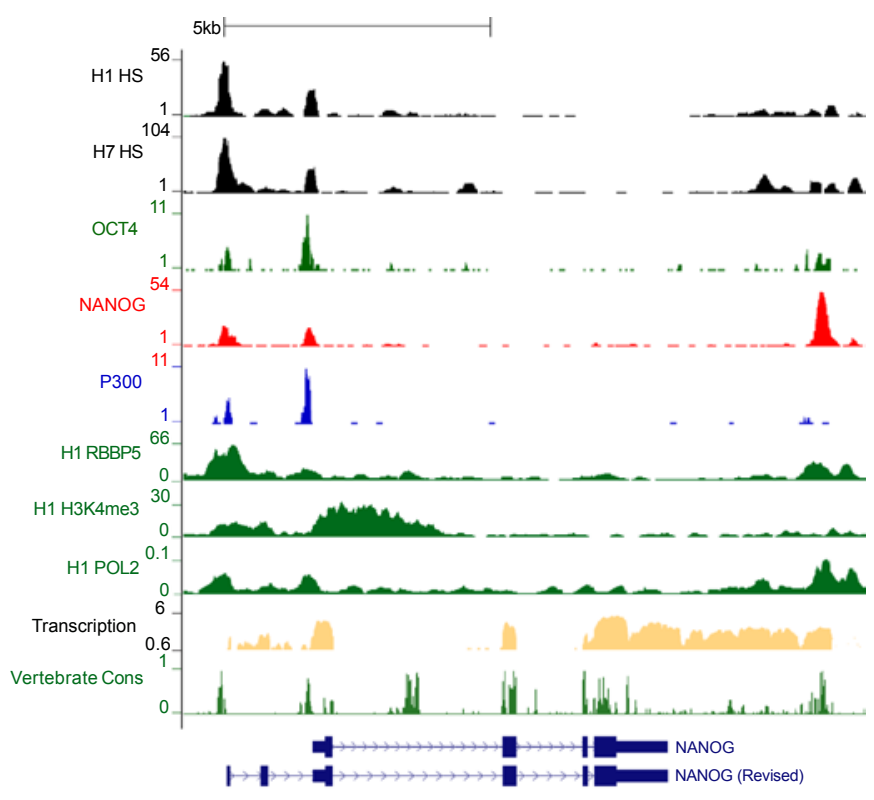

Figure 1: Transcriptional Regulation of NANOG in ESCs. NANOG gene structure is shown at the bottom with the revised 5' extended NANOG showing novel exons discovered recently in NTERA-2 cells [1]. NANOG regulatory regions are shown with DNAsel hypersensitivity peaks in $\mathrm{H} 1$ and $\mathrm{H} 7$ human ESCs. Chromatin occupancy profiles for ESC transcription factors (OCT4, NANOG), components of the basal transcriptional machinery (POL2 and p300) are shown at the NANOG locus in ESCs. The active chromatin mark $\mathrm{H} 3$ lysine 4 trimethylation ( $\mathrm{H} 3 \mathrm{~K} 4 \mathrm{me} 3$ ) profile and transcriptional expression levels generated by sequencing of polyA RNA are also depicted.

were designed in exon 1 (see revised NANOG gene structure suggested by Eberle et al. [32] and shown in Figure 1) and to compare against total NANOG transcript levels, primers were designed spanning the junction of exon 3 and exon 4 . The qPCR results confirmed the presence of NANOG transcripts originating from the upstream region (Figure 2). As we had observed previously in mouse ESCs, the stoichiometric ratio was skewed toward transcripts from the downstream TSS. This was verified by two independent primer pairs (Figure 2).

\section{Alternate promoters regulate the expression of NANOG}

The existence of NANOG transcripts originating from a region upstream of the known TSS implied that this location must house a second promoter. The RNA polymerase II ChIP-Seq data (Figure 1) also revealed binding peaks at the upstream region suggesting RNA polymerase II recruitment to the upstream promoter. To explore and identify the second promoter, we amplified human genomic DNA regions upstream of NANOG exon 1 which is highly conserved among vertebrate sequences analyzed (Figure 3B). Different DNA fragments of the NANOG upstream region were used as promoters to drive expression of the neomycin resistance $\left(\mathrm{Neo}^{\mathrm{R}}\right)$ gene and form colonies in a chromatinized stable transfection assay that we employed previously to analyze murine Nanog regulatory elements [36]. The proximal promoter $(-258$ to +34$)$ was used to compare the strength of the two promoter regions. The human NANOG proximal promoter has been shown to be highly active in mouse ESCs [33]; and unlike human ESCs, these cells are amenable to the $\mathrm{Neo}^{\mathrm{R}}$ single cell colony assay. For these reasons, we performed the $\mathrm{Neo}^{\mathrm{R}}$ assay in mouse J1 ESCs. High numbers of neomycin resistant colonies were obtained from cells transfected with either the proximal (P1) or the distal (P2) promoters in comparison to cells transfected with a Hoxb6 promoter containing plasmid (Figure 3C and 3D). These results disclose a novel promoter (P2) upstream of the recently discovered exon 1 [32]. Although the proximal P1 promoter produced a significant number of neomycin resistant colonies in comparison to the control; surprisingly, P2 is much stronger than P1 in this assay. A minimal promoter consisting of the sequence between -1788 to -1737 produced the highest number of neomycin-resistant colonies. Analysis of this sequence by TRANSFAC predicted an abundance of transcription factor binding elements, including TFIID, CBP, CEBP alpha and NF-I in this short region (Figure $3 \mathrm{~A}$ ). This minimal promoter also has two well conserved predicted Nanog binding sites that are likely candidates for the significant NANOG occupancy we observe at this region. Additional DNA sequences at either end of the minimal promoter diminished the activation capacity of the minimal promoter. Inclusion of the Alu SINE (short interspersed element) upstream (NANOG -2401 to -1702) of the minimal promoter completely abolished the activation potential of the promoter revealing the inhibitory capacity of the SINE. Additionally, the minimal $\mathrm{P} 2$ promoter (-1788 to -1737) was orientation-independent as the sequence in reverse orientation also produced similar number of colonies. This orientation independence explains why the mouse counterpart of this regulatory element may have been categorized previously as an enhancer by our group and others $[35,43,44]$. These data reveal a novel and highly active promoter in ESCs which regulates the expression of the upstream NANOG transcripts.

\section{Nanog regulates the proximal promoter in ESCs}

Among the ESC core transcription factor triad it is well established that Oct 4 and Sox 2 are involved in the transcriptional regulation of Nanog $[33,45,46]$; however, the contribution Nanog makes in the regulation of its own promoter remains unexplored. To determine if Nanog employs an autoregulatory mechanism to control its expression we scanned the NANOG P1 for the presence of the most conserved tetramer ATTA/C reported to be present at the center of Nanog binding sites $[15,25]$ and identified five such sites inside the P1

A

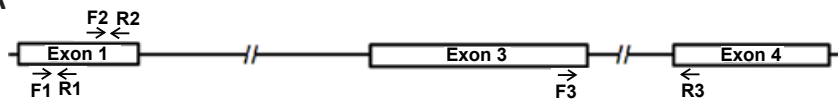

B

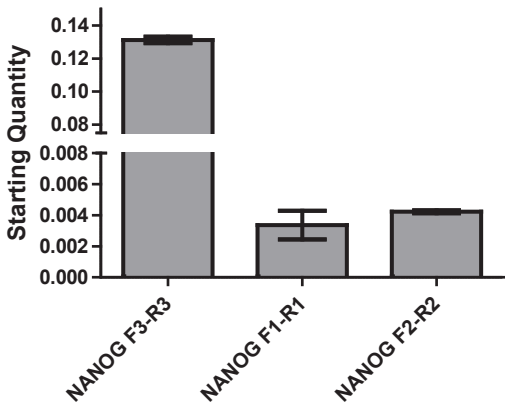

Figure 2: Novel NANOG transcripts in human ESCs. (A) A schematic of the updated NANOG gene structure showing the location of the two primer pairs used to detect the exon 1 originating transcripts. The F3-R3 primer pair hybridizing to exons 3 and 4 , respectively, was used to measure the total expression levels of total NANOG. qRT-PCR was used to measure the expression levels of total and novel NANOG transcripts initiating from the upstream exon 1 using RNA isolated from human $\mathrm{H} 13$ ESCs. The expression of upstream NANOG transcription is verified by two independent primer pairs. Levels shown are relative to a GAPDH control. 


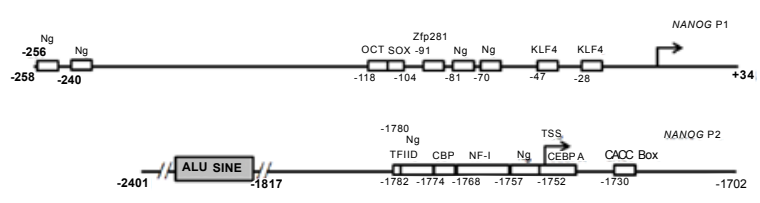

B

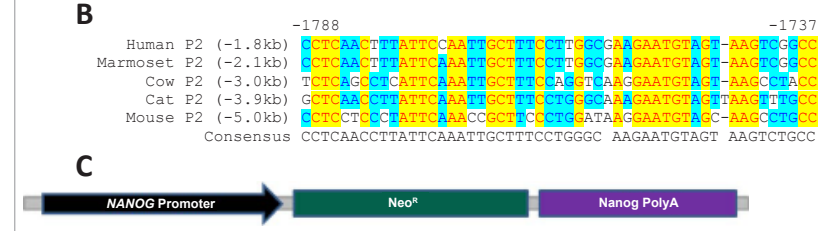

D

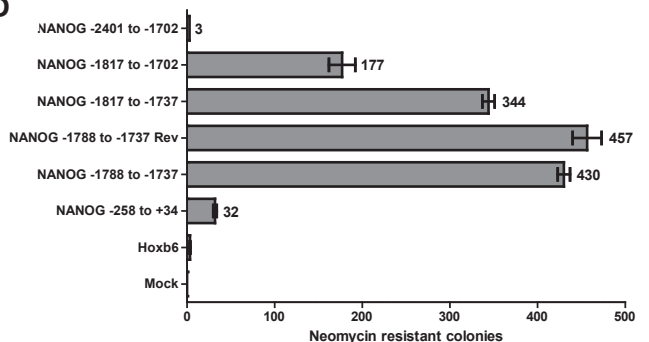

Figure 3: Neomycin resistance assay reveals alternate NANOG promoters in ESCs. (A) Schematic of the two NANOG promoters P1 (proximal promoter, upper) and P2 (distal promoter, below) showing the transcription factor binding sites in rectangular boxes with their respective positions to the TSS. KLF4, OCT4 and SOX2 are validated sites, whereas the NANOG binding sites are predicted by the presence of the tetramer ATTA/C. P2 shows transcription factor binding sites that are predicted by TRANSFAC. (B) Alignment of the upstream P2 promoter in mouse, cat, cow, marmoset and human showing that the NANOG P2 is moving closer to the TSS across evolutionary time. (C) A schematic of the plasmid showing the design used for the neomycin resistance assay. (D) Neomycin resistant colonies produced by the respective promoters are plotted. Control cells were mock-transfected and the Hoxb6 promoter was used as a negative control as it is not active in ESCs.

sequence $(-258$ to +34$)$, including one that bridged the sense strand of the Oct4-Sox2 tandem binding site. We wish to note here that a well evolutionarily conserved consensus TATA box (TATAAA) [47] exists at +3 bp according to the Genbank TSS (NM 024865.2, July 2012) that was annotated from NTERA-2 expressed sequence tags, including the cDNA originally deposited by Shinya Yamanaka (Accession number AK022643) [48]. The mouse and cow TSS, determined from ESCs and blastocysts, respectively, is placed at the exact expected position $32 \mathrm{bp}$ downstream of this TATA box (see NM 028016.2, June 2012 and NM 001025344.1, March 2012). We designed our P1 promoter based on this information and anticipate that future determination of the TSS in human ESCs using 5' amplification of cDNA ends (RACE) will result in the updating of our P1 coordinates to -291 to +1 . We employed a luciferase reporter assay to evaluate the contribution of presumptive NANOG ATTA/C binding sites in the regulation of P1 activity in CJ7 ESCs. In all predicted Nanog binding sites, ATT was mutated to CCG in the conserved tetramer to ablate Nanog binding. To disrupt the site hidden inside Oct4-Sox2 (ttttgcatTacaatg, predicted Nanog binding site underlined), only the $\mathrm{T}$ spacer nucleotide joining the Oct4 and Sox 2 binding sites was mutated to A to ensure that the Oct 4 and Sox 2 binding sites remained functional. NANOG P1 induced high-levels of luciferase expression. Out of the five predicted Nanog binding sites tested, mutations at sites between -256 to $-249,-240$ to -233 and the site between Oct4-Sox 2 binding had no effect on luciferase expression (data not shown). However, mutation of the sites at -81 to -74 (Nanog site-1) and -60 to -63 (Nanog site-2) resulted in $56 \%$ and $22 \%$ reductions in luciferase expression (Figure 4B) compared to the intact P1. Mutating both the sites resulted in a 59\% reduction in the P1 luciferase activity. A sequence alignment of this Nanog promoter region from seven vertebrates shows that along with the Oct4-Sox 2 binding cis-regulatory elements, the Nanog binding sites are also perfectly conserved throughout mammalian evolution (Figure 4A).

\section{Nanog binds to the NANOG proximal promoter}

After observing the reduction in NANOG P1 activity resulting from Nanog binding site mutagenesis we performed EMSAs to verify the ability of Nanog to bind these sites in vitro. Two different DNA fragments encompassing the Nanog binding sites were synthesized (Figure 5A) and radio-labeled. Nuclear extracts prepared from CJ7 ESCs resulted in shifts with both the probes. However, the binding was stronger at the Nanog site-2 containing probe in comparison to the site1 (Figure 5A). These bindings were competed off with excess unlabeled oligonucleotide. In contrast, oligonucleotides with the mutated Nanog binding site failed to compete as expected. Incubation of the nuclear extracts with mutant probes also did not result in the shift obtained by wild-type probes. These data show that the Nanog binding sites recruit Nanog and imply that this recruitment contributes to the activation of NANOG expression that we observe.

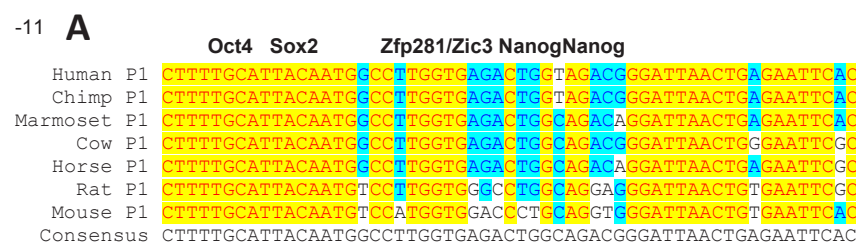

B

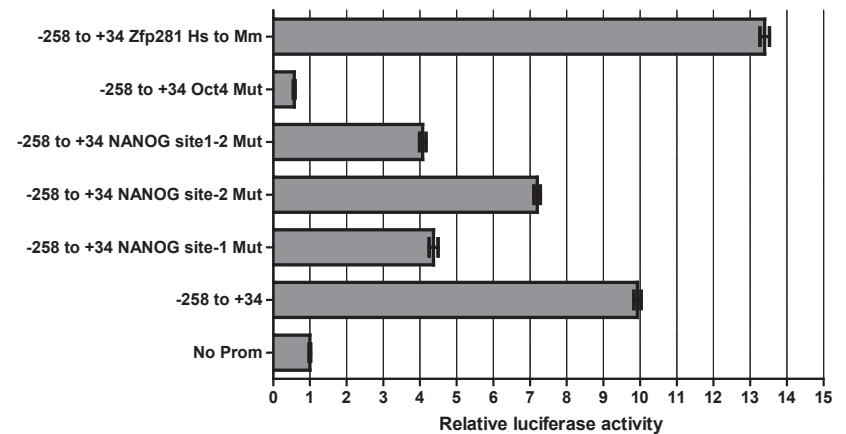

Figure 4: Nanog autoregulation of promoter P1. (A) An alignment of NANOG sequences from seven vertebrates in the region -119 to -63 relative to the human TSS. Oct4, Sox 2 and the Nanog binding sites are perfectly conserved and indicated by solid bars, whereas the Zfp281/Zic3 site by a wavy line. The human (Hs) Zfp281/Zic3 site was mutagenized to produce a consensus Zic3/Zfp281 mouse site $(\mathrm{Mm})$ by mutating CTGGTAG to CCTGCAG. (B) Luciferase assay was performed in CJ7 ESCs. Cells were transfected with the NANOG promoter reporter constructs (left) and analyzed for promoter activity. The Oct4 binding site was mutated GCAT to AACC and used as a positive control to show ablation of promoter activity. The Nanog binding sites were mutated from ATT to CCG at the conserved tetramer ATTA/C. Firefly luciferase expression levels were normalized to the luciferase activity of internal Renilla control. The no promoter containing plasmid (No Prom) was used as the internal control and its activity was normalized to 1 . Data presented are the mean \pm SEM of triplicates from one of two independent experiments. 


\section{A NgP1-WT1: Gtggtagacgggattaactgagaatt NgP1-WT1-Mut: GtggtagacgggCCtaactgagaatt NgP1-WT2: Gttaactgagaattcacaagggtgg NgP1-WT2-Mut: GttaactgagaCCtcacaagggtgg}

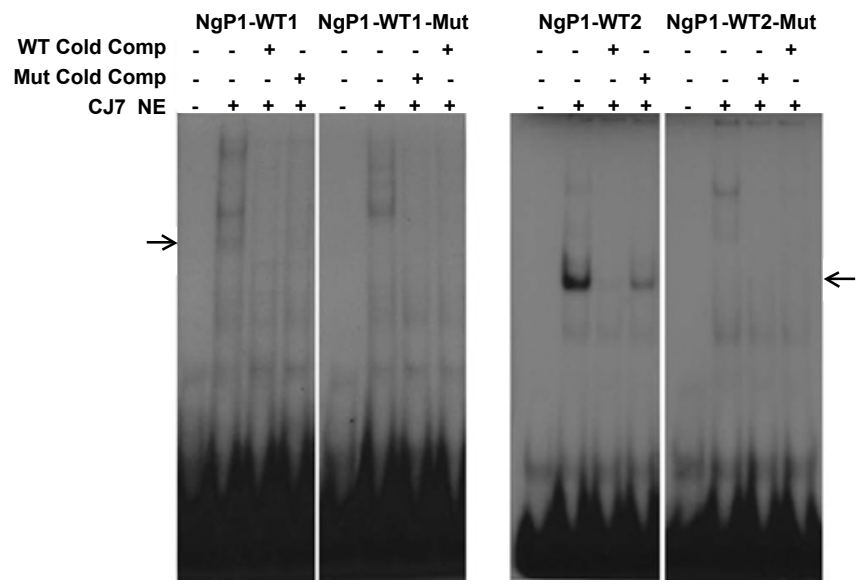

Figure 5: Nanog binds to the Nanog-binding sites in the human NANOG promoter. (A) Sequence of the Nanog motif (underlined) containing NANOG proximal promoter oligonucleotides used as probe. Mutant (Mut) oligonucleotides have their binding motifs disrupted as shown in capital letters. Oligonucleotides were annealed to their antisense strands and labeled with $\left[\mathrm{Y}^{-32} \mathrm{P}\right] \mathrm{dCTP}$ using Klenow by filling the $\mathrm{G}$ at both ends of the probe. Unlabeled oligonucleotides of the WT and Mut sequences were used for competitive binding. Binding was tested using nuclear extracts of CJ7 ES cells. Protein-DNA complexes of the resulting from Nanog binding is shown by arrow mark in the WT probes, whereas these shifts were not observed with the mutant probes. Unlabeled mutant oligonucleotides were also not able to compete off the binding resulting from the WT probes. (B) Sequence of the Zfp281 motif (underlined) restored to the mouse sequence of the Nanog promoter. Shift observed by incubation of the Zfp281-site restored probe incubation with CJ7 nuclear extract (indicated by arrow) was not observed by the human WT probe. The unlabeled human WT probe was also unable to compete off the shift resulting from the mutant probe.

\section{Zfp281 binding site in the NANOG P1 promoter is lost during evolution}

Analysis of the Nanog P1 promoter sequence alignment revealed that the mouse Zfp281 binding site [49] is not well conserved in the human NANOG P1 (Figure 4A). This site is also bound by the pluripotency factor Zic3 as demonstrated by Nanog promoter activation in ESCs [50]. To determine the effect of $\mathrm{Zfp} 281$ binding to this site we restored the mouse site (ctggtag to cCTgCag) by site-directed mutagenesis of the human P1. Luciferase reporter assay showed that restoring the mouse Zfp281 site enhanced the luciferase expression by $35 \%$ over the wild-type human P1. To verify Zfp281 binding to the restored mouse site in human P1 we performed EMSAs. DNA fragments containing either the wild-type human $\mathrm{P} 1$ or a 3 basepair alteration that restores the mouse Zfp281 binding footprint were synthesized and radiolabeled for use as EMSA probes (Figure 5B). Incubation of the probes with CJ7 nuclear extracts resulted in a shift with the probe harboring a restored mouse Zfp281 binding site and this was not observed with the wild-type human P1 probe. EMSA in presence of excess unlabeled oligonucleotides showed that while the mouse Zfp281 binding-site restored probe was able to compete off the binding, the wild-type probe failed to do so. These results demonstrate that the Zfp281 binding site in human P1 is lost during the course of mammalian evolution and that this contributes to a dampening of NANOG P1 activation.

\section{Discussion}

The unique self-renewal and pluripotency characteristics of ESCs are regulated by a multi-layered network of post-transcriptional and post-translational modifications [51]. Alternate splicing augments this regulatory control by increasing transcriptome and proteome diversity from a single gene locus. The resulting additional protein isoforms may contribute to the maintenance of the ESC state or commitment to different lineage specification by modifying the protein interaction network or by localizing to different compartment of the cell. Alternately, changes in non-coding sequences may alter posttranscriptional regulation. Alternative splicing points to alternate promoter selection and the activation or inhibition of different promoters for the same gene may impact lineage commitment and differentiation. The core pluripotency factor OCT4 is known code for multiple transcripts and protein variants, out of which only OCT4A is implicated for pluripotency of ESCs, whereas the other versions, OCT4B and OCT4B1 are expressed in more differentiated cell types [52,53]. Our previous studies in mouse ESCs revealed multiple transcripts of Nanog capable of coding for two additional protein isoforms with differential capacities for maintaining a pluripotency gene signature [23]. In the present study, we verified the transcription of NANOG from a novel upstream exon in human ESCs and identified a strong promoter upstream (P2) responsible for transcription initiating from this exon. Although there was a more robust DNAseI HS regulatory footprint at P2, we observed that there was a decreased enrichment of Oct 4 and p300 at this region that might affect assembly of a robust RNA transcriptional complex. Alternatively, transcripts may be produced with comparable strength at both $\mathrm{P} 1$ and $\mathrm{P} 2$, but diminished elongation of transcripts from P2 resulting from a less processive RNA polymerase complex or due to post-transcriptional regulatory mechanisms might contribute to the lower levels of fully processed transcripts produced from this alternate promoter. We also determined that Nanog employs a positive autoregulatory mechanism to control its own downstream promoter (P1).

The transcription factor Nanog functions collaboratively with the other core pluripotency factors Oct4 and Sox2 to govern the maintenance of pluripotency. Even though Nanog was originally shown to be critical largely for repressing primitive endoderm formation [25], work by others implies that it is responsible for repressing genes essential for germ layer specification in the embryo proper as well [54]. Using a system that enabled the controlled loss of Nanog function in ESCs, we recently demonstrated that Nanog functions as a global repressor of critical genes that underlie function of the early mesodermal, endodermal and ectodermal germ layer compartments, as well as primitive endoderm [23]. Nanog remains functionally unique among the core pluripotency triad for this global gene repression capacity. We were intrigued by this phenomenon and wished to determine if regulatory elements positioned near the Nanog gene might provide mechanistic insight on how Nanog represses genes responsible for lineage specification. By analyzing HS that marked the Nanog promoters in adult cells and organs of mice and humans, we revealed strong regulatory element footprints in tissues (Figure 6). Intriguingly, when a 5712 bp DNA cassette containing both Nanog promoters is inserted into the beta globin locus that is permissive for expression only in erythroid cells, DNA methylation levels reveal that both regulatory elements remain active in ESCs [55]. This suggests that Nanog regulatory elements are sufficient to remodel a normally repressive chromatin domain and make it amenable for gene expression. In contrast, upon differentiation into neural progenitor cells, the upstream promoter region remains unmethylated and active 
A

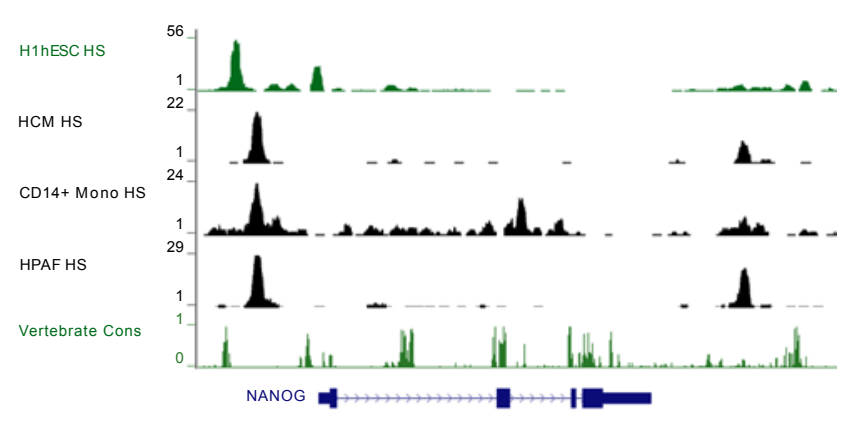

B

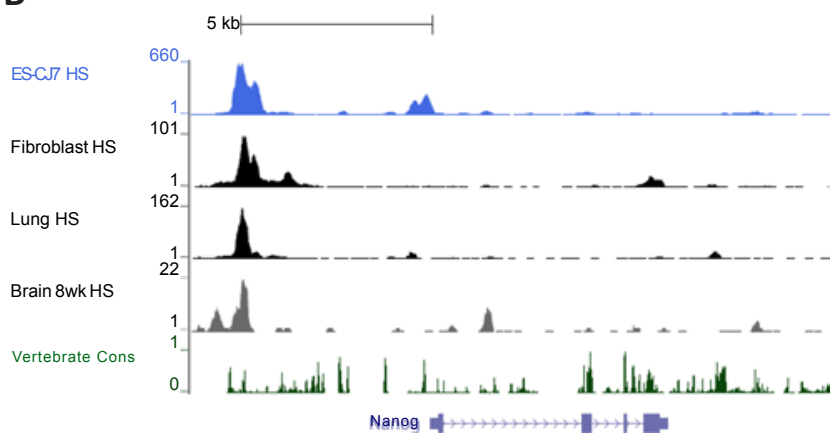

Figure 6: NANOG promoter P2 sequence conservation and DNAsel HS at the NANOG locus in different tissues. (A) NANOG gene structure is shown at the bottom. DNAsel HS peaks in H1 ESCs, human cardiomyocytes (HCM), CD14 monocytes and human pancreatic adenocarcinoma cells (HPAF) showing the active cis-regulation mark at the upstream P2 promoter persists in adult differentiated cells, whereas the HS mark observed in the ESCs at the proximal P1 promoter is extinguished in the adult cells. (B) A similar persistence of the HS peak at P2 is observed in mouse adult cells while the downstream $\mathrm{P} 1$ peak is extinguished.

but the downstream promoter is completely extinguished. This implies that the upstream alternate promoter harbors regulatory elements that enable it to remain permissive for gene expression in pluripotent and adult cells. Previous work has suggested that genes of certain cellular lineages may be marked in pluripotent cells by a histone signature that poises gene expression to be turned on later during lineage specification in a well-defined spatial and temporal manner [56]. Conversely, this Nanog regulatory element may serve as a platform to mark a potent repression footprint that ensures that certain Nanog protein variant(s) are not expressed in adult lineages. Compelling hints have suggested that Nanog may function in adult tissues [57-61]. For this reason, we cannot rule out the possibility that Nanog alternative transcripts expressed from the upstream promoter may encode proteins that function to regulate tissues in post-embryonic stem and progenitor cell populations.

An alignment of the most active human $\mathrm{P} 2$ region (-1788 to -1737 , Figure $3 \mathrm{~B}$ ) showed that the sequence is well conserved among vertebrates. However NANOG $\mathrm{P} 1$ and $\mathrm{P} 2$ are narrowing a genomic gap during evolution as the distance of $5 \mathrm{~kb}$ in the mouse has gradually contracted down to $1.8 \mathrm{~kb}$ in the human gene locus. The sequence analysis of this region in TRANSFAC predicted binding sites of potent components of the basal transcriptional machinery. The inclusion of TFIID, CBP, CEBPA and NF-1 (Figure 3A) binding footprints within this short sequence might explain the significant strength of this small promoter in comparison to the proximal P1 promoter (Figure 3C). Two ATTA/C sites predicting NANOG binding motifs are also present in this short sequence. The upstream site is on the sense strand, the downstream site lies on the antisense strand, and this Nanog tandem likely confers strength upon this promoter. One of these sequences has also been experimentally validated as a Nanog binding site in mouse ESCs [15].

While NANOG binding to the NANOG proximal promoter has been demonstrated [41], the regulatory effect of this binding has not been analyzed previously. Utilizing site-directed mutagenesis and EMSAs we identified the Nanog binding sites in the proximal promoter and showed that Nanog contributes to the activation of its own promoter. Nanog is known to exist as a homodimer in ESCs [36]. Given the proximity of the two Nanog binding motifs in P1, it is possible that Nanog binds to these sites through a monomeric or homodimeric configuration.

The human P1 resulted in expression of neomycin in our stable transfection assay; however, the promoter strength measured by this assay was significantly weaker in comparison to the P2 minimal promoter (Figure 3C). Additionally, a mouse P1 of near identical size was significantly stronger than the human P1 (unpublished observations). Sequence alignment of the conserved P1 sequences showed that while the Oct4-Sox2 and Nanog binding sites are well conserved in the $\mathrm{P} 1$ sequence, a binding site for the pluripotency factor Zfp281 is lost in the human promoter (Figure 4A). Zfp281 has been shown to activate the mouse P1 [49] by binding to this site. Another pluripotency factor Zic3 has also been shown to activate the mouse P1 by binding to the same region [50]. When we restored the human P1 sequence to match the mouse binding site, it resulted in a significant increase in human P1 activity (Figure 4B). Zfp281 is known to interact with Nanog [7,36]. In the absence of Zfp281, Nanog recruitment to the Nanog site in mouse P1 was shown to be significantly diminished in the absence of Zfp281 in ESCs [62]. Perhaps in the absence of an intact Zfp281 site in NANOG P1, efficient Nanog recruitment is diminished. As we have shown that Nanog activates P1 activity, less efficient binding could explain its loss of strength.

\section{Conclusion}

The discovery of an alternate NANOG promoter that functions in ESCs significantly expands the regulatory repertoire used to control this core pluripotency factor. A comprehensive study of the human NANOG locus in human ESCs will be required in the future to reveal the full upstream exon structure and to determine if additional protein variants exist, as suggested by our recent work in mouse ESCs [23]. The existence of a potent alternate promoter positioned at the 5 ' end of the gene locus presents an additional mechanism for dampening expression of the predominant NANOG transcript and protein through the production of upstream open reading frames (uORFs) [63]. Alternative transcripts that we discovered in mouse ESCs exhibit loss or gain of large uORFs [23] that frequently serve to sequester the translational apparatus away from downstream coding sequences. Future work will be required to determine if the human upstream NANOG promoter serves a dual role in producing novel protein variants and downregulating the predominant NANOG A protein.

\section{Acknowledgement}

This work was supported in part by a Basil O'Connor Starter Scholar Research Award Grant (5-FY10-45) from the March of Dimes Foundation. 
Citation: Das S, Jena S, Kim EM, Zavazava N, Levasseur DN, et al. (2012) Transcriptional Regulation of Human NANOG by Alternate Promoters in Embryonic Stem Cells. J Stem Cell Res Ther S10:009. doi:10.4172/2157-7633.S10-009

\section{References}

1. Evans MJ, Kaufman MH (1981) Establishment in culture of pluripotential cells from mouse embryos. Nature 292: 154-156.

2. Martin GR (1981) Isolation of a pluripotent cell line from early mouse embryos cultured in medium conditioned by teratocarcinoma stem cells. Proc Natl Acad Sci USA 78: 7634-7638.

3. Smith AG (2001) Embryo-derived stem cells: of mice and men. Annu Rev Cell Dev Biol 17: 435-462.

4. Takahashi K, Yamanaka S (2006) Induction of pluripotent stem cells from mouse embryonic and adult fibroblast cultures by defined factors. Cell 126: 663-676.

5. Yu J, Vodyanik MA, Smuga-Otto K, Antosiewicz-Bourget J, Frane JL, et al (2007) Induced pluripotent stem cell lines derived from human somatic cells. Science 318: 1917-1920.

6. Hanna J, Wernig M, Markoulaki S, Sun CW, Meissner A, et al. (2007) Treatment of sickle cell anemia mouse model with iPS cells generated from autologous skin. Science 318: 1920-1923.

7. Wang J, Rao S, Chu J, Shen X, Levasseur DN, et al. (2006) A protein interaction network for pluripotency of embryonic stem cells. Nature 444: 364-368.

8. Boyer LA, Lee TI, Cole MF, Johnstone SE, Levine SS, et al. (2005) Core transcriptional regulatory circuitry in human embryonic stem cells. Cell 122 947-956.

9. Ivanova N, Dobrin R, Lu R, Kotenko I, Levorse J, et al. (2006) Dissecting selfrenewal in stem cells with RNA interference. Nature 442: 533-538.

10. Chen X, Xu H, Yuan P, Fang F, Huss M, et al. (2008) Integration of externa signaling pathways with the core transcriptional network in embryonic stem cells. Cell 133: 1106-1117.

11. Kim J, Chu J, Shen X, Wang J, Orkin SH, et al. (2008) An extended transcriptional network for pluripotency of embryonic stem cells. Cell 132: 1049-1061.

12. van den Berg DL, Snoek T, Mullin NP, Yates A, Bezstarosti K, et al. (2010) An Oct4-centered protein interaction network in embryonic stem cells. Cell Stem Cell 6: 369-381.

13. Brandenberger R, Wei H, Zhang S, Lei S, Murage J, et al. (2004) Transcriptome characterization elucidates signaling networks that control human ES cell growth and differentiation. Nat Biotechnol 22: 707-716.

14. Wei CL, Miura T, Robson P, Lim SK, Xu XQ, et al. (2005) Transcriptome profiling of human and murine ESCs identifies divergent paths required to maintain the stem cell state. Stem Cells 23: 166-185.

15. Loh YH, Wu Q, Chew JL, Vega VB, Zhang W, et al. (2006) The Oct4 and Nanog transcription network regulates pluripotency in mouse embryonic stem cells. Nat Genet 38: 431-440

16. Barash Y, Calarco JA, Gao W, Pan Q, Wang X, et al. (2010) Deciphering the splicing code. Nature 465: 53-59.

17. Kunarso G, Wong KY, Stanton LW, Lipovich L (2008) Detailed characterization of the mouse embryonic stem cell transcriptome reveals novel genes and intergenic splicing associated with pluripotency. BMC Genomics 9: 155.

18. Pritsker M, Doniger TT, Kramer LC, Westcot SE, Lemischka IR (2005) Diversification of stem cell molecular repertoire by alternative splicing. Proc Natl Acad Sci USA 102: 14290-14295

19. Salomonis N, Schlieve CR, Pereira L, Wahlquist C, Colas A, et al. (2010) Alternative splicing regulates mouse embryonic stem cell pluripotency and differentiation. Proc Natl Acad Sci USA 107: 10514-10519.

20. Yeo GW, Xu X, Liang TY, Muotri AR, Carson CT, et al. (2007) Alternative splicing events identified in human embryonic stem cells and neural progenitors. PLoS Comput Biol 3: 1951-1967.

21. Gabut M, Samavarchi-Tehrani P, Wang X, Slobodeniuc V, O'Hanlon D, et al. (2011) An alternative splicing switch regulates embryonic stem cell pluripotency and reprogramming. Cell 147: 132-146.

22. Wu JQ, Habegger L, Noisa P, Szekely A, Qiu C, et al. (2010) Dynamic transcriptomes during neural differentiation of human embryonic stem cells revealed by short, long, and paired-end sequencing. Proc Natl Acad Sci USA 107: 5254-5259.
23. Das S, Jena S, Levasseur DN (2011) Alternative splicing produces Nanog protein variants with different capacities for self-renewal and pluripotency in embryonic stem cells. J Biol Chem 286: 42690-42703.

24. Rao S, Zhen S, Roumiantsev S, McDonald LT, Yuan GC, et al. (2010) Differential roles of Sall4 isoforms in embryonic stem cell pluripotency. Mol Cell Biol 30: 5364-5380.

25. Mitsui K, Tokuzawa Y, Itoh H, Segawa K, Murakami M, et al. (2003) The homeoprotein Nanog is required for maintenance of pluripotency in mouse epiblast and ES cells. Cell 113: 631-642.

26. Chambers I, Colby D, Robertson M, Nichols J, Lee S, et al. (2003) Functiona expression cloning of Nanog, a pluripotency sustaining factor in embryonic stem cells. Cell 113: 643-655.

27. Wang SH, Tsai MS, Chiang MF, Li H (2003) A novel NK-type homeobox gene ENK (early embryo specific NK), preferentially expressed in embryonic stem cells. Gene Expr Patterns 3: 99-103.

28. Hart AH, Hartley L, Ibrahim M, Robb L (2004) Identification, cloning and expression analysis of the pluripotency promoting Nanog genes in mouse and human. Dev Dyn 230: 187-198.

29. Hyslop L, Stojkovic M, Armstrong L, Walter T, Stojkovic P, et al. (2005) Downregulation of NANOG induces differentiation of human embryonic stem cells to extraembryonic lineages. Stem Cells 23: 1035-1043.

30. Zaehres H, Lensch MW, Daheron L, Stewart SA, Itskovitz-Eldor J, et al. (2005) High-efficiency RNA interference in human embryonic stem cells. Stem Cells 23: 299-305

31. Darr H, Mayshar Y, Benvenisty N (2006) Overexpression of NANOG in human ES cells enables feeder-free growth while inducing primitive ectoderm features. Development 133: 1193-1201.

32. Eberle I, Pless B, Braun M, Dingermann T, Marschalek R (2010) Transcriptiona properties of human NANOG1 and NANOG2 in acute leukemic cells. Nucleic Acids Res 38: 5384-5395.

33. Kuroda T, Tada M, Kubota H, Kimura H, Hatano SY, et al. (2005) Octame and Sox elements are required for transcriptional cis regulation of Nanog gene expression. Mol Cell Biol 25: 2475-2485.

34. Chan KK, Zhang J, Chia NY, Chan YS, Sim HS, et al. (2009) KLF4 and PBX1 directly regulate NANOG expression in human embryonic stem cells. Stem Cells 27: $2114-2125$

35. Levasseur DN, Wang J, Dorschner MO, Stamatoyannopoulos JA, Orkin SH (2008) Oct4 dependence of chromatin structure within the extended Nanog locus in ES cells. Genes Dev 22: $575-580$

36. Wang J, Levasseur DN, Orkin SH (2008) Requirement of Nanog dimerization for stem cell self-renewal and pluripotency. Proc Natl Acad Sci USA 105: 63266331.

37. Das S, Lin D, Jena S, Shi A, Battina S, et al. (2008) Protection of retinal cells from ischemia by a novel gap junction inhibitor. Biochem Biophys Res Commun 373: 504-508.

38. Kim J, Cantor AB, Orkin SH, Wang J (2009) Use of in vivo biotinylation to study protein-protein and protein-DNA interactions in mouse embryonic stem cells Nat Protoc 4: 506-517.

39. ENCODE Project Consortium (2011) A user's guide to the encyclopedia of DNA elements (ENCODE). PLoS Biol 9: e1001046.

40. Ram O, Goren A, Amit I, Shoresh N, Yosef N, et al. (2011) Combinatoria patterning of chromatin regulators uncovered by genome-wide location analysis in human cells. Cell 147: 1628-1639.

41. Kunarso G, Chia NY, Jeyakani J, Hwang C, Lu X, et al. (2010) Transposable elements have rewired the core regulatory network of human embryonic stem cells. Nat Genet 42: 631-634.

42. Lister R, Pelizzola M, Dowen RH, Hawkins RD, Hon G, et al. (2009) Human DNA methylomes at base resolution show widespread epigenomic differences. Nature 462: 315-322.

43. Wu Q, Chen X, Zhang J, Loh YH, Low TY, et al. (2006) Sall4 interacts with Nanog and co-occupies Nanog genomic sites in embryonic stem cells. J Bio Chem 281: 24090-24094.

44. Kagey MH, Newman JJ, Bilodeau S, Zhan Y, Orlando DA, et al. (2010) Mediator and cohesin connect gene expression and chromatin architecture. Nature 467: 430-435. 
Citation: Das S, Jena S, Kim EM, Zavazava N, Levasseur DN, et al. (2012) Transcriptional Regulation of Human NANOG by Alternate Promoters in Embryonic Stem Cells. J Stem Cell Res Ther S10:009. doi:10.4172/2157-7633.S10-009

45. Rodda DJ, Chew JL, Lim LH, Loh YH, Wang B, et al. (2005) Transcriptional regulation of nanog by OCT4 and SOX2. J Biol Chem 280: 24731-24737.

46. Wu da Y, Yao Z (2005) Isolation and characterization of the murine Nanog gene promoter. Cell Res 15: 317-324.

47. Bucher P (1990) Weight matrix descriptions of four eukaryotic RNA polymerase II promoter elements derived from 502 unrelated promoter sequences. J Mol Biol 212: 563-578.

48. Booth HA, Holland PW (2004) Eleven daughters of NANOG. Genomics 84 229-238.

49. Wang ZX, Teh $\mathrm{CH}$, Chan CM, Chu C, Rossbach M, et al. (2008) The transcription factor Zfp281 controls embryonic stem cell pluripotency by direct activation and repression of target genes. Stem Cells 26: 2791-2799.

50. Lim LS, Hong FH, Kunarso G, Stanton LW (2010) The pluripotency regulator Zic3 is a direct activator of the Nanog promoter in ESCs. Stem Cells 28: 19611969.

51. Young RA (2011) Control of the embryonic stem cell state. Cell 144: 940-954.

52. Wang X, Zhao Y, Xiao Z, Chen B, Wei Z, et al. (2009) Alternative translation of OCT4 by an internal ribosome entry site and its novel function in stress response. Stem Cells 27: 1265-1275.

53. Atlasi Y, Mowla SJ, Ziaee SA, Gokhale PJ, Andrews PW (2008) OCT4 spliced variants are differentially expressed in human pluripotent and nonpluripotent cells. Stem Cells 26: 3068-3074.

54. Hatano SY, Tada M, Kimura H, Yamaguchi S, Kono T, et al. (2005) Pluripotential competence of cells associated with Nanog activity. Mech Dev 122: 67-79.

55. Lienert F, Wirbelauer C, Som I, Dean A, Mohn F, et al. (2011) Identification of genetic elements that autonomously determine DNA methylation states. Nat Genet 43: 1091-1097.

56. Xu J, Watts JA, Pope SD, Gadue P, Kamps M, et al. (2009) Transcriptional competence and the active marking of tissue-specific enhancers by defined transcription factors in embryonic and induced pluripotent stem cells. Genes Dev 23: 2824-2838.

57. Bais MV, Shabin ZM, Young M, Einhorn TA, Kotton DN, et al. (2012) Role of Nanog in the maintenance of marrow stromal stem cells during post natal bone regeneration. Biochem Biophys Res Commun 417: 211-216.

58. Hasmim M, Noman MZ, Lauriol J, Benlalam H, Mallavialle A, et al. (2011) Hypoxia-dependent inhibition of tumor cell susceptibility to CTL-mediated lysis involves NANOG induction in target cells. J Immunol 187: 4031-4039.

59. Kohler EE, Cowan CE, Chatterjee I, Malik AB, Wary KK (2011) NANOG induction of fetal liver kinase-1 (FLK1) transcription regulates endothelial cel proliferation and angiogenesis. Blood 117: 1761-1769.

60. Kucia M, Reca R, Campbell FR, Zuba-Surma E, Majka M, et al. (2006) A population of very small embryonic-like (VSEL) CXCR4(+)SSEA-1(+)Oct-4+ stem cells identified in adult bone marrow. Leukemia 20: 857-869.

61. Lee TK, Castilho A, Cheung VC, Tang KH, Ma S, et al. (2011) CD24(+) liver tumor-initiating cells drive self-renewal and tumor initiation through STAT3mediated NANOG regulation. Cell Stem Cell 9: 50-63.

62. Fidalgo M, Shekar PC, Ang YS, Fujiwara Y, Orkin SH, et al. (2011) Zfp281 functions as a transcriptional repressor for pluripotency of mouse embryonic stem cells. Stem Cells 29: 1705-1716.

63. Calvo SE, Pagliarini DJ, Mootha VK (2009) Upstream open reading frames cause widespread reduction of protein expression and are polymorphic among humans. Proc Natl Acad Sci USA 106: 7507-7512.
This article was originally published in a special issue, Embryonic and Induced Pluripotent Stem Cells handled by Editor(s). Dr. Jianlong Wang, Mount Sinai School of Medicine, United States 